\title{
Atividade antioxidante de Piper arboreum, Piper dilatatum e Piper divaricatum
}

SILVA, J.A.'; OLIVEIRA, F.F.'; GUEDES, E.S.'; BITTENCOURT, M.A.L.2; OLIVEIRA, R.A. ${ }^{*}$

Universidade Estadual de Santa Cruz, Departamento de Ciências Exatas e Tecnológicas; 'Universidade Estadual de Santa Cruz, Departamento de Ciências Agrárias e Ambientais; Rodovia Jorge Amado, km-16, Bairro Salobrinho, Ilhéus-Bahia, Brasil, 45652-900. *rosilene@uesc.br

RESUMO: Os óleos essenciais de P. arboreum, P. dilatatum e P. divaricatum foram obtidos por hidrodestilação e analisados por CG-DIC e CG-EM. Extratos etanólicos foram preparados por extração exaustiva. A atividade antioxidante de óleos e extratos foi avaliada por meio do método de sequestro de radicais livres usando 2,2-difenil-1-picril-hidrazila. Os teores de óleos essenciais foram de $0,98 \%, 1,50 \%$ e $0,99 \%$ para $P$. arboreum, $P$. dilatatum e $P$. divaricatum, respectivamente. Esses óleos demonstraram riqueza em sesquiterpenos, sendo os principais componentes: biciclogermacreno (28,7\%) e $\beta$-copaen-4- $\alpha$-ol $(13,3 \%)$ para $P$. arboreum; germacreno $D(16,7 \%)$, $\alpha$-alaskeno (18,9\%) e viridiflorol (12,5\%) para $P$. dilatatum; e germacreno D $(9,4 \%)$, valenceno $(11,1 \%)$ e $\gamma$-cadineno $(11,0 \%)$ para $P$. divaricatum. No teste de atividade antioxidante, com base nas percentagens de sequestro de radicais, foram determinados a concentrações efetivas $\left(\mathrm{CE}_{50}\right)$ e o Índice de Atividade Antioxidante (IAA). Os seguintes valores de $C_{50}$ e IAA foram encontrados: ácido ascórbico (usado como referência) $226,84 \mu \mathrm{g} \cdot \mathrm{mL}^{-1} \mathrm{e}$ 5,30; extrato de $P$. arboreum 239,60 $\mu \mathrm{g} \cdot \mathrm{mL}^{-1}$ e 4,90 , e extrato de $P$. dilatatum $367,70 \mu \mathrm{g} \cdot \mathrm{mL}^{-1}$ e 3,20, respectivamente. A metodologia utilizada para a atividade antioxidante mostrou-se inadequada para o extrato da $P$. divaricatum. Os óleos essenciais não apresentaram atividade antioxidante significativa, entretanto, os extratos etanólicos de $P$. arboreum e de $P$. dilatatum apresentaram atividade antioxidante expressiva.

Palavras-chave: Piperaceae, DPPH, óleo essencial, extrato etanólico

\begin{abstract}
Antioxidant activity of Piper arboreum, Piper dilatatum, and Piper divaricatum. The essential oils of $P$. arboreum, $P$. dilatatum and $P$. divaricatum were obtained by means of hydrodistillation and analyzed by GC-FID and GC-MS. The ethanolic extracts were prepared by exhaustive extraction. The antioxidant activity of the oils and extracts were evaluated by applying the free radical scavenging method using the 2,2-diphenyl-1-picrylhydrazyl radical. The yields of the essential oils were $0.98 \%, 1.50 \%$ and $0.99 \%$ for $P$. arboreum, $P$. dilatatum and $P$. divaricatum, respectively. The oils are rich in sesquiterpenes, and the main components of $P$. arboreum are the bicyclogermacrene (28.7\%) and $\beta$-copaen-4- $\alpha-\mathrm{ol}(13.3 \%)$; of $P$. dilatatum, the germacrene D (16.7\%), a-alaskene (18.9\%) and viridiflorol (12.5\%); and of $P$. divaricatum, the germacrene $D(9.4 \%)$, valencene $(11.1 \%)$ and $\gamma$-cadinene $(11.0 \%)$. The antioxidant activity test, based on the percentages of radical scavenging, determined the effective concentrations $\left(C_{50}\right)$ and the Antioxidant Activity Index (AAI). The following $C_{50}$ and AAI values were found: $226.84 \mu \mathrm{g} \cdot \mathrm{mL}^{-1}$ and 5.30 for ascorbic acid (used as the reference), $239.60 \mu \mathrm{g} \cdot \mathrm{mL}^{-1}$ and 4.90 for $P$. arboreum, and $367.70 \mu \mathrm{g} \cdot \mathrm{mL}^{-1}$ and 3.20 for $P$. dilatatum. The antioxidant evaluation using this methodology is not applicable for the $P$. divaricatum extract. These essential oils did not present a significant antioxidant activity. However, the ethanolic extracts of $P$. arboreum and $P$. dilatatum did show a strong antioxidant activity.
\end{abstract}

Key words: Piperaceae, DPPH, essential oil, ethanolic extract

\section{INTRODUÇÃO}

Piper arboreum Aubl. var arboreum, Piper dilatatum Rich. e Piper divaricatum G. Mey. pertencem a família Piperaceae que abrange 12 gêneros com aproximadamente 2.000 espécies. Piper é o maior gênero dessa família com cerca de 700 espécies, e dessas, 266 têm ocorrência no 
Brasil (Yuncker, 1972). Muitas espécies de Piper são aromáticas e seus óleos essenciais são constituídos de monoterpenos, sesquiterpenos, fenilpropanóides, aldeídos, cetonas e alcoóis de cadeia longa (Cysne et al., 2005). Algumas espécies são grandes produtoras de óleos essenciais (Maia et al., 1997), e alguns extratos vegetais apresentam aplicações medicinais, propriedades inseticidas, bactericidas e fungicidas (Potzernheim et al., 2006; Nascimento, 2011).

Piper arboreum conhecida popularmente como pau-de-angola, jaborandi, pimenta do mato, é um arbusto glabro de altura entre 2 a 4 metros, de ocorrência da América Central até o Sul do Brasil. É utilizada como anti-reumática, sudorífera, afrodisíaca e emoliente (Guimarães \& Monteiro, 2006).

Piper dilatatum é um arbusto com 1,5 a 3 metros de altura encontrada na região sul da Bahia. Tem propriedades antifúngicas (Pohlit et al., 2004), e suas folhas têm ações antiinflamatória e cicatrizante, aliviando crises de bronquite e asma, enquanto as raízes são usadas no tratamento de odontalgias (Andrade et al., 2009).

Piper divaricatum, conhecida popularmente também como pau-de-angola, jaborandi-manso, "beetle" é um arbusto com até 9 metros de altura encontrada em vários estados brasileiros incluindo a região sul da Bahia. As folhas e raízes, quando cozidas, são usadas para banhos antirreumáticos e, em infusão, são empregadas internamente contra dores reumáticas e cólicas. A raiz apresenta cheiro forte (Andrade et al., 2009).

Os óleos essenciais de $P$. arboreum e $P$. dilatatum apresentam considerável atividade moluscida contra Biomphalaria glabrata, atividades citotóxica, inseticida, fungicida e antimicrobiana (Santos et al., 2001; Constantin et al., 2001).

Extratos de espécies vegetais podem apresentar diversas atividades biológicas merecendo destaque a atividade antioxidante. As substâncias capazes de atuar como antioxidante, quando presentes em concentrações ideais em relação aos substratos oxidáveis, inibem ou retardam significativamente os processos oxidativos (Vaya \& Aviram, 2001) e protegem os sistemas biológicos dos danos produzidos por doenças degenerativas e câncer (Souza et al., 2007). Devido a essas propriedades, muitas espécies têm despertado o interesse das indústrias farmacêutica, alimentícia e cosmética. Os antioxidantes naturais, devido aos aspectos tecnológicos e nutricionais, têm sido estudados para substituir os antioxidantes sintéticos (Pitaro et al., 2012).

Para determinar a atividade antioxidante in vitro, tem sido utilizado o método baseado na mudança de coloração de uma solução composta por radicais estáveis 2,2-difenil-1-picrilhidrazila (DPPH), de cor violeta, que adicionado às substâncias que podem ceder um átomo de hidrogênio mudam de coloração. Esse método é rápido, simples e estável, tornando-se facilmente reprodutível (Huang et al., 2005; Duarte-Almeida et al., 2006; Kuskoski et al., 2006).

Exemplos do emprego desse método são os estudos com os frutos in natura de açaí, acerola, morango e amora que apresentam atividade antioxidante muito forte. Merece destaque a acerola que, por ter altas concentrações de ácido ascórbico, apresenta atividade pró-oxidante verificada pelo método que utiliza $\beta$-caroteno e ácido linoleico. Essa característica não pode ser determinada pelo método do DDPH (DuarteAlmeida et al., 2006). Extratos etanólicos das folhas, cascas e raizes das plantas medicinais Terminalia brasiliensis, Terminalia fagifolia, Cenostigma macrophyllum, Qualea grandiflora e Copernicia prunifera apresentaram atividade antioxidante (Sousa et al., 2007). Essa ação também foi observada com as tinturas de Hamamelis virginiana, Psidium guajava, Própolis, Matricaria chamomilla e Hydrocotyle asiatica (Vicentino \& Menezes, 2007). Extratos etanólicos das folhas de Baccharis dracunculifolia e Taraxacum officinale e das inflorescências de Bidens segetum apresentam significativa atividade antioxidante (Fabri et al., 2011).

Poucos são os trabalhos envolvendo o estudo da atividade antioxidante de óleos essenciais e extratos etanólicos de Piperaceas. Esse trabalho visou determinar a composição química dos óleos essenciais dessas três espécies de Piper, de ocorrência espontânea na região de Una (Bahia), e o potencial antioxidante de óleos e extratos etanólicos utilizando o método de seqüestro de radicais livres com DPPH.

\section{MATERIAL E MÉTODO}

\section{Coleta do material vegetal}

As folhas de $P$. arboreum, $P$. dilatatum e $P$. divaricatum foram coletadas no Distrito de Una, Bahia, no período da manhã em agosto de 2011. Exsicatas das espécies foram depositadas no Herbário da Universidade Estadual de Santa Cruz (HUESC) sob os números 14.101, 14.104 e 14.102, respectivamente, e identificadas por Luiz Alberto Mattos da Silva, Elsie Franklin Guimarães e José Lima da Paixão. As folhas foram submetidas ao processo de secagem, através da utilização de estufa (DE LEO) com ventilação forçada a uma temperatura média de $50^{\circ} \mathrm{C}$, até obter peso

Rev. Bras. PI. Med., Campinas, v.16, n.3, supl. I, p.700-706, 2014. 
constante.

\section{Extração dos óleos essenciais}

As extrações foram feitas com material vegetal seco e a técnica utilizada foi a hidrodestilação utilizando-se aparelho Clevenger (Craveiro et al., 1981). Cerca de 30,0g do material seco foi extraído por 120 minutos para a $P$. arboreum e $P$. dilatatum e 150 minutos para $P$. divaricatum. O óleo essencial foi separado do hidrolato usando diclorometano, tratado com sulfato de sódio anidro por 60 minutos e concentrado. As extrações foram feitas em triplicata. Os teores dos óleos foram determinados pela massa dos óleos, em triplicata, usando balança analítica, e expressos em porcentagem massa/massa (g de óleo por $100 \mathrm{~g}$ de matéria vegetal seca).

\section{Análise dos óleos essenciais}

As análises de cromatografia gasosa (CG) com detector de ionização de chama (DIC) foram realizadas em cromatógrafo a gás Varian Saturno 3800 com coluna capilar VF-5ms de (30m $X 0,25 \mathrm{~mm} \times 0,25 \mu \mathrm{m})$. Foi injetado $1 \mu \mathrm{L}$ da solução de óleo (10\%) em $\mathrm{CHCl}_{3}$. As condições de análise foram: temperatura do injetor: $250^{\circ} \mathrm{C}$, do detector: $280^{\circ} \mathrm{C}$, gás de arraste He com fluxo de $1,2 \mathrm{~mL} \cdot \mathrm{min}^{-1}$. A propagação da coluna teve início a $80^{\circ} \mathrm{C}$ por 2 minutos, seguido de acréscimo de $4^{\circ} \mathrm{C} \cdot \mathrm{min}^{-1}$, até atingir $140^{\circ} \mathrm{C}$, seguido de acréscimo de $20^{\circ} \mathrm{C} \mathrm{min}{ }^{-1}$, até $280^{\circ} \mathrm{C}$, sendo mantida nessa temperatura $5 \mathrm{~min}$.

As análises de espectrometria de massa (EM) foram realizadas no espectrofotômetro de massa Varian Saturno Chromopack 2000 MS/MS por impacto eletrônico de 70 eV, sendo a temperatura da transferline de $250^{\circ} \mathrm{C}$ e do trap $220^{\circ} \mathrm{C}$. A coluna e as condições de temperatura foram às mesmas usadas na análise CG-DIC. Os diversos constituintes químicos dos óleos essenciais foram identificados através da comparação computadorizada com a biblioteca do aparelho (NIST05 e NIST08), literatura e índice de retenção Kovatz (Adams, 2007). Os índices de retenção Kovatz (IK) foram calculados através da injeção de uma série de padrões de $n$-alcanos $\left(\mathrm{C}_{8}-\mathrm{C}_{26}\right)$ injetados nas mesmas condições cromatográficas das amostras.

\section{Extrato etanólico}

O extrato etanólico foi preparado por extração exaustiva (Matos, 1997) colocando em um erlenmeyer $10 \mathrm{~g}$ da folha seca e triturada em $100 \mathrm{~mL}$ de etanol. Após o período de 48h, com agitação ocasional, foi realizada a filtração a vácuo. $\mathrm{O}$ extrato foi concentrado e o material vegetal foi novamente submetido ao processo de extração. 0 mesmo procedimento foi repetido cinco vezes e as amostras foram agrupadas.

\section{Atividade antioxidante}

A técnica constituiu em adicionar $1 \mathrm{~mL}$ de solução metanólica 0,2 mM do radical DPPH, mantida sob refrigeração e na ausência de luz, a 2,5 mL de cada uma das soluções do óleo essencial e extrato etanólico nas concentrações de 5, 50, 125, 250 e $500 \mu \mathrm{g} \cdot \mathrm{mL}^{-1}$ e ao padrão, ácido ascórbico. Para cada amostra foi feito o controle negativo com metanol. As soluções foram mantidas por 30 minutos no escuro à temperatura ambiente. Após este período foram feitas leituras das absorbâncias em comprimento de onda de 517 nm no espectrofotômetro UV/VIS Thermo Scientifc. A amostra controle foi preparada com 2,5 mL do solvente e $1 \mathrm{~mL}$ da solução de DPPH, sem adição de antioxidante. Todas as análises foram realizadas em triplicata. Como referência foi utilizado o ácido ascórbico (AAS). A porcentagem de sequestro de radicais livres (\%SRL) foi calculada correlacionando-se a absorbância média de cada amostra com a absorbância da solução controle ao final da reação conforme Equação 1. A concentração efetiva $\left(\mathrm{CE}_{50}\right)$ foi calculada a partir regressão linear obtida do gráfico da concentração das amostras e o restante de SRL, que corresponde a diferença entre o valor máximo e a quantidade de SRL encontrada, nas diferentes concentrações estudadas. O índice de atividade antioxidante (IAA) foi calculado correlacionando-se a massa de DPPH $\left(\mu \mathrm{g} \cdot \mathrm{mL}^{-1}\right)$ com $\mathrm{CE}_{50}\left(\mu \mathrm{g} \cdot \mathrm{mL}^{-1}\right)$ conforme Equação 2:

$\%$ SRL: $\underline{A b s}_{\underline{c}}-\mathrm{Abs}_{\underline{a}} \times 100$

Abs $_{c}$

Equação 1: Abs $_{c}$ : Absorção da solução controle; Abs: Absorção da amostra

IAA: $\underline{m}_{\underline{D P H}}$

$\mathrm{CE}_{50}$

Equação 2: $\mathrm{m}_{\mathrm{DPPH}}$ : massa de DPPH; $\mathrm{CE}_{50}$ : concentração efetiva

\section{Análise estatística}

No teste antioxidante foi aplicada a análise de variância (ANOVA) com comparação de médias pelo teste de Tukey a 5\% de significância.

\section{RESULTADO E DISCUSSÃO}

As análises dos óleos essenciais por cromatografia gasosa acoplada à espectrometria de massa permitiram identificar 41 constituintes químicos distribuídos entre $P$. arboreum, $P$. dilatatum e $P$. divaricatum (Tabela 1 ), sendo predominantes sesquiterpenos e sesquiterpenos oxigenados. Os componentes $\gamma$-muuroleno, espatulenol e germacreno $D$ foram comuns às três espécies.

O teor de óleo essencial de $P$. arboreum foi de $0,98 \pm 0,02 \%$ e foram identificados $89,9 \%$ dos compostos, distribuídos em $59,2 \%$ de

Rev. Bras. Pl. Med., Campinas, v.16, n.3, supl. I, p.700-706, 2014. 
sesquiterpenos e $30,7 \%$ de sesquiterpenos oxigenados. Os principais componentes majoritários foram biciclogermacreno $(28,7 \%)$ e $\beta$-copaen-4- $\alpha$-ol $(13,3 \%)$. Relatos na literatura mostram diferentes composições químicas para o óleo essencial dessa espécie de ocorrência em outros locais. Na Mata Atlântica (Rio de Janeiro) P. arboreum apresentou $\gamma$-eudesmol (14,6\%), $\alpha$-eudesmol $(12,2 \%)$ e bulnesol $(8,1 \%)$ como componentes majoritários (Potzernheimet et al., 2006), enquanto na região da Amazônia (Amapá) isvarano (44,0\%) foi o único constituinte majoritário (Andrade et al., 2009). A subespécie Piper arboreum arboreum da região do Cerrado (Distrito Federal) apresentou como componentes majoritários biciclogermacreno $(12,1 \%)$, óxido de cariofileno $(10,2 \%)$ e $10-e p i-\gamma$ eudesmol (Potzernheimet et al., 2006).

O teor de óleo essencial em $P$. dilatatum foi de $1,50 \pm 0,01 \%$ e foram identificados $84,2 \%$ dos compostos, apresentando $50,7 \%$ de sesquiterpenos e $33,5 \%$ de sesquiterpenos oxigenados. Os três principais componentes majoritários foram germacreno D (16,7\%), $\alpha$-alaskeno $(18,9 \%)$ e viridiflorol (12,5\%). Estudos da composição química de óleos essenciais de $P$. dilatatum mostraram grandes variações intraespecífica entre indivíduos procedentes do Cerrado (Distrito Federal) e da Mata Atlântica (Rio de Janeiro). No Cerrado os componentes majoritários identificados foram cis- $\beta$-ocimeno (19,6\%), $\beta$-cariofileno $(11,3 \%)$ e espatulenol $(6,5 \%)$ e na Mata Atlântica $\alpha$-pipeno $(17,7 \%), \alpha$-felandreno $(41,8 \%)$ e 1,8 -cineol $(2,7 \%)$ (Potzernheimet et al., 2006). No Pará, essa espécie apresentou como constituintes majoritários $\alpha$-pipeno (9,7\%), $\beta$-pipeno (14,8\%), (Z)- $\beta$-Ocimeno $(10,0 \%)$, biciclogermacreno $(27,6 \%)$ e espatulenol $(15,0 \%)$ (Andrade et al., 2009). A avaliação da composição dos óleos essenciais de doze espécimes coletadas no leste da Amazônia mostrou grande variabilidade de componentes majoritários, diferindo dos óleos extraídos de outras Piperaceas de ocorrência em outras localidades da Amazônia, que normalmente apresentam como componente majoritário fenilpropanóides (Andrade et al., 2011).

Comparando os dados da literatura com o presente estudo não foi observada semelhança entre os constituintes químicos.

Em $P$. divaricatum o teor de óleo foi de $0,99 \pm 0,07 \%$. A composição química foi complexa sendo identificados apenas $67,4 \%$ dos constituintes, classificados em $46,7 \%$ de sesquiterpenos e $20,7 \%$ de sesquiterpenos oxigenados. Os componentes majoritários foram germacreno $\mathrm{D}(9,4 \%)$, valenceno $(11,1 \%), \gamma$-cadineno $(11,0 \%)$. Em outro espécime de ocorrência na serra de Guaramiranga (Ceará) observou-se $\beta$-pineno (19,3\%), linalool (16,5\%) e $\alpha$-pineno $(13,6 \%)$ como constituintes majoritários
(Almeida et al., 2009). No óleo essencial de outro espécime, coletado no Estado da Amazônias, os componentes majoritários foram $\beta$-cariofileno $(21,1 \%)$, óxido de cariofileno (16,6\%), $\beta$-eudesmol $(8,7 \%)$ e $\alpha$-eudesmol (Andrade et al., 2009). O espécime proveniente de Itabuna Itabuna (Bahia) fornece um óleo essencial rico em safrol, diferindo desse estudo (Barbosa et al., 2012), embora haja proximidade entre as localidades.

A composição química dos óleos das espécies estudadas neste trabalho difere dos dados da literatura, sugerindo grande susceptibilidade desses metabólitos secundários aos fatores bióticos e abióticos (Simões, Spitzer; 2003).

A atividade antioxidante foi avaliada por meio do método de sequestro de radicais livres usando o radical 2,2-difenil-1-picril-hidrazila (DPPH). A partir da percentagem de sequestro de radicais ( $S R L)$, Tabela 2 , foram determinadas as concentrações efetivas $\left(\mathrm{CE}_{50}\right)$ e o Índice de Atividade Antioxidante (IAA), usando-se ácido ascórbico (AAS) como referência (Tabela 3).

Segundo Fabri e colaboradores (2011), quanto menor $\mathrm{o}$ valor do $\mathrm{CE}_{50}$ para a substância, maior será a atividade antioxidante. $O$ extrato etanólico de $P$. arboreum apresentou atividade similar ao AAS enquanto os óleos apresentaram atividade antioxidante muito fraca. $\mathrm{ACE}_{50}$ representa a concentração necessária para inibir $50 \%$ da concentração inicial do DPPH, e, no caso específico do extrato etanólico da $P$. divaricatum, mesmo na menor concentração, $5 \mu \mathrm{g} \cdot \mathrm{mL}^{-1}$, foi observada inibição de $70 \%$ da atividade sequestradora de radicais. Dessa forma, o valor gerado pelo método da regressão linear para a $\mathrm{CE}_{50}$ seria negativo, incoerente, considerando a definição de concentração. No entanto, ao considerar que quanto menor $\mathrm{O}$ valor de $\mathrm{CE}_{50}$ maior será a atividade antioxidante sugerimos que esse extrato pode apresentar atividade pró-antioxidante, porém, essa propriedade não é detectada utilizando-se o método do DPPH (Duarte-Almeida et al., 2006).

Segundo Scherer e colaboradores (2009) através do valor do IAA é possível classificar a ação antioxidante como muito fraca (IAA $<0,5)$, fraca (IAA entre 0,5 e 1,0), moderada (IAA entre 1,0 a 2,0), forte (IAA entre 2,0 e 2,5) e muito forte (IAA > 2,5). Portanto, os extratos etanólicos de $P$. arboreum e $P$. dilatatum apresentaram ação antioxidante muito forte, enquanto os óleos apresentaram ação muito fraca. (Tabela 3) Esse resultado para os óleos essenciais pode ser atribuído as suas composições químicas, nas quais predominam sesquiterpênicos, classe de compostos que não apresentam capacidade sequestradora de radicais.

Os extratos, hidroalcoólico e em acetato de etila, das folhas de $P$. arboreum, coletada em São

Rev. Bras. PI. Med., Campinas, v.16, n.3, supl. I, p.700-706, 2014. 
TABELA 1. Composição química dos óleos essenciais extraídos das folhas secas de $P$. arboreum, $P$. dilatatum e P. divaricatum

\begin{tabular}{|c|c|c|c|c|c|c|c|c|c|}
\hline $\mathrm{IK}^{*}$ & Componentes & $\begin{array}{c}P . \\
a r b\end{array}$ & $\begin{array}{l}P . \\
\text { dil }\end{array}$ & $\begin{array}{c}P . \\
\text { div }\end{array}$ & $\mathrm{IK}^{*}$ & Componentes & $\begin{array}{c}P . \\
a r b\end{array}$ & $\begin{array}{l}P . \\
\text { dil }\end{array}$ & P. div \\
\hline 1379 & a-copaeno & 0,7 & & 4,3 & 1553 & germancreno B & 6,0 & & \\
\hline 1390 & $\beta$-cubebeno & 1,8 & & & 1553 & $\begin{array}{c}\beta \text {-calacoreno } \\
\text { hidrato de cis- }\end{array}$ & & 0,7 & \\
\hline 1393 & $\beta$-elemeno & & 5,1 & & 1556 & sesquisabineno & & & 1,7 \\
\hline 1425 & (E)-cariofileno & 8,6 & & 0,8 & 1563 & ledol & & 3,8 & \\
\hline 1430 & Aromadendreno & 1,3 & 1,4 & & 1574 & espatulenol & 0,6 & 1,1 & 3,5 \\
\hline 1450 & Seicheleno & & 0,7 & & 1586 & $\beta$-copaen-4- $\alpha$-ol & 13,3 & & \\
\hline 1460 & a-humuleno & 1,2 & & & 1590 & viridiflorol & 6,4 & 12,5 & \\
\hline 1466 & allo-aromadendreno & 0,7 & & & 1593 & óxido de cariofileno & & & 6,5 \\
\hline 1467 & 9-epi-(E)-cariofileno & & 0,9 & & 1600 & kusimone & & 1,6 & \\
\hline 1471 & $\gamma$-himachaleno & & 1,6 & & 1600 & guaiol & 2,1 & & \\
\hline 1475 & germacreno D & 4,6 & 16,7 & 9,4 & 1611 & 1,10-di-epi-cubenol & & 1,3 & 1,3 \\
\hline 1477 & $\gamma$-muuroleno & 1,2 & 2,0 & 8,3 & 1635 & a-acorenol & 1,7 & & \\
\hline 1492 & cis- $\beta$-guaieno & 2,1 & & & 1636 & 1-epi-cubenol & & & 2,5 \\
\hline 1491 & Valenceno & & & 11,1 & 1642 & cubenol & & 1,2 & 1,3 \\
\hline 1496 & Biciclogermacreno & 28,7 & & & 1642 & himachalol & 2,3 & & \\
\hline 1498 & Curzereno & & 3,8 & & 1649 & cedra-8 (15)-en-9-a-ol & & 2,1 & \\
\hline 1510 & $\alpha$-alaskeno & & 18,9 & & 1652 & valerianol & 1,2 & & 3,9 \\
\hline 1513 & $\gamma$-cadineno & & & 11,0 & 1658 & 7-epi-a-eudesmol & 2,3 & 1,8 & \\
\hline 1517 & 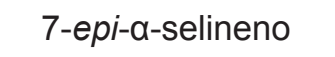 & 2,1 & & & 1690 & kusinol & 0,9 & 2,4 & \\
\hline 1521 & $\delta$-cadineno & & 2,7 & & 1691 & acetato de cariofileno & & 2,1 & \\
\hline 1524 & cis-calamenese & & & 1,7 & & Total identificado (\%) & 89,9 & 84,2 & 67,4 \\
\hline
\end{tabular}

P. arb: Piper arboreum; P. dil: Piper dilatatum; P.div: Piper divaricatum. IK*: Índice kovats experimental: listados em ordem de eluição em coluna HP-5ms e determinado com referência a série homóloga de alcanos $\left(\mathrm{C}_{8}-\mathrm{C}_{26}\right)$. Percentagens calculadas pelo método de normatização das áreas. 
TABELA 2. Porcentagem de sequestro de radicais livres dos óleos essenciais e extratos etanólicos de $P$. arboreum, P. dilatatum e P. divaricatum

\begin{tabular}{ccccccccc}
\hline \multirow{2}{*}{$\begin{array}{c}\text { Concentrações } \\
\mu \mathrm{g} \cdot \mathrm{mL}^{-1}\end{array}$} & \multicolumn{2}{c}{$P$. arboreum } & \multicolumn{2}{c}{ P. dilatatum } & \multicolumn{2}{c}{ P. divaricatum } & \multirow{2}{*}{ AAS } \\
\cline { 2 - 6 } 500 & O.E. & Ext. Et. & O.E. & Ext. Et. & O.E. & Ext. Et. & \\
\hline & $4,4^{\mathrm{a}}$ & $92,2^{\mathrm{a}}$ & $7,5^{\mathrm{a}}$ & $62,6^{\mathrm{a}}$ & $4,4^{\mathrm{a}}$ & $95,7^{\mathrm{a}}$ & & $95,4^{\mathrm{a}}$ \\
250 & $4,1^{\mathrm{b}}$ & $92,0^{\mathrm{b}}$ & $6,5^{\mathrm{b}}$ & $41,3^{\mathrm{b}}$ & $3,9^{\mathrm{b}}$ & $95,5^{\mathrm{b}}$ & $95,2^{\mathrm{b}}$ \\
125 & $0,0^{\mathrm{c}}$ & $91,1^{\mathrm{c}}$ & $5,2^{\mathrm{c}}$ & $22,2^{\mathrm{c}}$ & $3,8^{\mathrm{c}}$ & $95,1^{\mathrm{c}}$ & $95,1^{\mathrm{c}}$ \\
50 & $0,0^{\mathrm{c}}$ & $87,6^{\mathrm{d}}$ & $4,7^{\mathrm{d}}$ & $14,9^{\mathrm{d}}$ & $3,1^{\mathrm{d}}$ & $94,9^{\mathrm{d}}$ & $33,2^{\mathrm{d}}$ \\
5 & $0,0^{\mathrm{c}}$ & $22,0^{\mathrm{e}}$ & $0,0^{\mathrm{e}}$ & $2,1^{\mathrm{e}}$ & $0,0^{\mathrm{e}}$ & $71,0^{\mathrm{e}}$ & & $0,0^{\mathrm{e}}$ \\
\hline
\end{tabular}

AAS: ácido ascórbico; O.E.: óleo essencial; Ext. Et.: extrato etanólico. Porcentagem de sequestro de radicais determinados através da média de leitura de três absorções para cada concentração. Letras iguais na mesma coluna significam que não houve variância. $\mathrm{O}$ teste de Tukey foi realizado para comparação de média.

TABELA 3. Valores de concentração efetiva e índice de atividade antioxidante dos óleos essenciais e extratos etanólicos de $P$. arboreum, $P$. dilatatum e $P$. divaricatum

\begin{tabular}{cccccc}
\hline \multirow{2}{*}{ Espécies } & \multicolumn{2}{c}{$\mathrm{CE}_{50}$} & \multicolumn{2}{c}{ IAA } \\
\cline { 2 - 6 } & O.E. & Ext. Et. & O.E. & Ext. Et. \\
\hline P. arboreum & 4.916 & 239,60 & 0,24 & 4,90 \\
P. dilatatum & 4.118 & & 367,70 & 0,29 & 3,20 \\
P. divaricatum & 5.622 & NA & 0,21 & NA \\
\hline AAS & \multicolumn{2}{c}{226,84} & & \multicolumn{2}{c}{5,20} \\
\hline
\end{tabular}

AAS: ácido ascórbico; O.E.: óleo essencial; Ext. Et.: extrato etanólico; $\mathrm{CE}_{50}$ : concentração efetiva; IAA: índice de atividade antioxidante; NA: Não se aplica

Paulo, exibiram atividade antioxidante moderada (Regasini et al., 2008) diferindo do extrato etanólico da espécie estudada nesse trabalho

No presente estudo os extratos etanólicos das folhas secas de $P$. arboreum e $P$. dilatatum apresentaram atividade antioxidante muito forte, ao contrário dos óleos essenciais; estas atividades devem-se, provavelmente, as diferentes composições dos metabolitos secundários presentes nos diferentes materiais vegetais.

\section{AGRADECIMENTOS}

A Fundação de Amparo a pesquisa do Estado da Bahia pelo suporte financeiro. A UESC pela concessão de bolsa de iniciação cientifica e ao Prof. Alex-Alan Furtado pelo suporte nas analises cromatográficas.

\section{REFERÊNCIAS}

ADAMS, R.P. Identification of Essential Oil Components by Gas Chromatography/Quadrupole Mass Spectroscopy. 4.ed. Illinois USA: Allured Publishing Corporation: Carol Stream, 2007. 804p.
ANDRADE, E.H.A.; GUIMARAES, E.F.; MAIA, J.G.S. Variabilidade química em óleos essenciais de espécies de Piper da Amazônia. 22.ed. Belém: FEQ/ UFPA, 2009. 448p.

ANDRADE, E.H.A.; ALVES, C.N.; GUIMARÃES, E.F.; CARREIRA, L.M.M.; MAIA, J.G.S. Variability in essential oil composition of Piper dilatatum L. C. Rich. Biochemical Systematics and Ecology, v.39, p.669675, 2011.

ALMEIDA, J.G.L. de.; SILVEIRA, E.R.; PESSOA, O.D.L.; NUNES, E.P. Essential oil composition from leaves and frits of Piper divaricatum G. Mey. Journal of Essencial Oil Research, v.21, n.3, p.228-230, 2009.

BARBOSA, Q.P.S.; CÂMARA, C.A.G.; RAMOS, C.S.; NASCIMENTO, D.C.O.; LIMA-FILHO, J.V.; GUIMARÃES, E.F. Chemical composition, circadian rhythm and antibacterial activity of essential oils of Piper divaricatum: a new source of safrole. Química Nova, v.35, n.9, p.1806-1808, 2012.

CRAVEIRO A.A.; FERNANDES A.G.; ANDRADE C.H.S.; MATOS F.J.A.; ALENCAR J.W.;

MACHADO M.I.L. Óleos essenciais de Plantas do Nordeste. Fortaleza: UFC, 1981. 210p.

CYSNE, J.B.; CANUTO, K.M.; PESSOA, O.D.L.; NUNES, E.P.; SILVEIRA, E.R. Leaf essential oils of four Piper species from the state of Ceará - northeast of Brazil. Journal of the Bazilian Chemical Society, v.16, n.6,

Rev. Bras. PI. Med., Campinas, v.16, n.3, supl. I, p.700-706, 2014. 
p.1378-1381, 2005.

CONSTANTIN, M.B.; SARTORELLI, P.; LIMBERGER, R.; HENRIQUES, A.T; ESTEPE, M.; FERREIRA, M.J.P.; OHARA, M.T.; EMERENCIANO, VP.; KATO, M.J. Essential Oils from Piper cernuum and Piper regnellii: Antimicrobial Activities and Analysis by GC/MS and ${ }^{13}$ C-NMR. Planta Medica, v.67, p.771-773, 2001.

DUARTE-ALMEIDA, J.M.; SANTOS, R.J.; GENOVESE, M.I.; LAJOLO, F.M. Avaliação da Atividade Antioxidante Utilizando Sistema $\beta$-caroteno/ácido linoléico e Método de Seqüestro de Radicais DPPH. Ciência e Tecnologia de Alimentos, v.26, n.2, p.446-452, 2006.

FABRI, R.L.; NOGUEIRA, M.S.; DUTRA, L.B.; BOUZADA, M.L.M., SCIO, E. Potencial antioxidante e antimicrobiano de espécies da família Asteraceae. Revista Brasileira de Plantas Medicinais, v.13, n.2, p.183-189, 2011.

GUIMARAES E.F \& MONTEIRO, D. Piperaceae na reserva biológica de Poço das Antas, Silva Jardim, Rio de Janeiro, Brasil. Rodriguésia, v.57, n.3, p.569-589. 2006.

HUANG, D.; OU, B.; PRIOR, R. The chemistry behind antioxidant capacity assays. Journal of Agriculturaland Food Chemistry, v.53, n.6, p.1.8411.856, 2005.

KUSKOSKI, E.M.; ASUERO, A.G.; MORALES, M.T.; FETT R. Frutos tropicais silvestres e polpas de frutas congeladas: atividade antioxidante, polifenóis e antocianinas. Ciência Rural, v.36, n.4, p.1283-1287, 2006.

MAIA, J.G.; ZOGHBI, M.G.; ANDRADE, E.H.A.; SANTOS, A.S. \& SILVA, M.H.L. Banco de dados das Plantas Aromáticas da Amazônia: Inventário das Espécies de Piper. Belém PA. Relatório Final à Academia Brasileira de Ciências: MPEG, 1997.

MATOS, F.J. Introdução a Fitoquímica Experimental. 2.ed. Fortaleza: Edições da UFC, 1997. 141p.

NASCIMENTO, K.M. Composição Química e Atividade Antifúngica dos Óleos Essenciais de Espécies de Piper Frente a Cepas de Candida SPP. 2011. 81p. Dissertação (Mestrado- Área de Concentração em Reprodução e Sanidade Animal) - Programa de PósGraduação em Ciências Veterinárias, Universidade Estadual do Ceará, Fortaleza.

PITARO, S.P.; FIORANI, L.V; JORGE, N. Potencial antioxidante dos extratos de manjericão (Ocimumbasilicum Laminaceae) e orégano (Origanum vulgare Laminaceae) em óleo de soja. Revista Brasileira de Plantas Medicinais, v.14, n.4, p.686691, 2012.

POHLIT, A.M.; QUIGNARD, E.L.J.; NUNOMURA, S.M.; TADEI, W.P.; HIDALGO, A. F. et al. Screening of plants found in the of Amazonas, Brazil for larvicidal activity against Aedes aegypti larvae. Acta amazonica, v.34, n.1, p.97-105, 2004.
POTZERNHEIM, M.C.L.; BIZZO, H.R.; VIEIRA, R.F. Análise dos óleos essenciais de três espécies de Piper coletadas na região do Distrito Federal (Cerrado) e comparação com óleos de plantas procedentes da região de Paraty, RJ (Mata Atlântica). Revista Brasileira de Farmacognosia, v.16, n.2, p.246-251, 2006.

REGASINI, L.O.; COTINGUIBA, F.; SIQUEIRA, J.R.; BOLZANI, V.S.; SILVA, D.H.S.; FURLAN, M.; KATO, M.J. Radical scavenging capacity of Piper arboreum and Piper tuberculatum (Piperaceae). Latin American Journal of Pharmacy (formerly Acta Farmacéutica Bonaerense), v.27, n.6, p.900-903, 2008.

SANTOS, P.R.D.; MOREIRA, D.E.; GUIMARÃES, E.F.; KAPLAN, M.A.C. Essential oil analysis of 10 Piperaceae species from the Brazilian Atlantic Forest. Phytochemistry, v.58, n.4, p.547-551, 2001.

SCHIO, E., FABRI, R.L.; NOGUEIRA, M.S.; DUTRA, L.B.; BOUZADA, M.L.M. Potencial antioxidante e antimicrobiano de espécies da família Asteraceae. Revista Brasileira de Plantas Medicinais, v.13, n.2, p.183-189, 2011.

SCHERER, R.; WAGNER, R.; DUARTE, M.C.T.; GODOY, H.T. Composição e atividade antimicrobiana dos óleos essenciais de cravo-da-índia, citronela e palmarosa. Revista Brasileira de Plantas Medicinais, v.11, n.4, p.442-449, 2009.

SIMÕES, C. M. O; SPITZER, V. Óleos voláteis. IN: SIMÕES, C. M. O et al. Farmacognosia : da planta ao medicamento. 5ed., Porto Alegre, Editora UFSC, 2003, p. 467-495.

SOUSA, C.M.M.; SILVA, H.R., VIEIRA-Jr G.M.; AYRES, M.C.C.; COSTA, C.L.S.; ARAÚJO, D.S.; CAVALCANTE, L.C.D.; BARROS, E.D.S.; ARAÚJO, P.B.M.; BRANDÃO, M.S.; CHAVES, M.H. Fenóis totais e atividade antioxidante de cinco plantas medicinais. Química Nova, v.30, n.2, p.351-355, 2007.

SOUZA, T.J.T.; APEL, M.A.; BORDIGNON, S.; MATZENBACHER N.I.; ZUANAZZI, J.A.S.; HENRIQUES, A.T. Composição química e atividade antioxidante do óleo volátil de Eupatorium polystachyum DC. Revista Brasileira de Farmacognosia, v.17, n.3, p.368-372, 2007.

VAYA J.; AVIRAM M. Nutritional antioxidants: mechanisms of action, analysis of activities and medical applications. Current Medicinal Chemistry - Immunology, Endocrine \& Metabolic Agents, v.1,n.1,p.99117, 2001.

VICENTINO, A.R.R.; MENEZES, F.S. Atividade antioxidante de tinturas vegetais, vendidas em farmácias com manipulação e indicadas para diversos tipos de doenças. Revista Brasileira de Farmacognosia, v.17, n.3, p.384-387, 2007.

YUNCKER, T.G. The Piperaceae of Brazil. I. Piper - Group I, II, III, IV. Hoehnea, v.2, p.19- 366, 1972.

Rev. Bras. PI. Med., Campinas, v.16, n.3, supl. I, p.700-706, 2014. 